médecine/sciences $1988 ; 8: 492-499$

\title{
La chorée de Huntington
}

La chorée de Huntington est une maladie autosomique dominante caractérisée par l'apparition progressive, habituellement entre 30 et 50 ans, de mouvements anormaux et d'une démence s'aggravant inexorablement. Le gène morbide est localisé à l'extrémité du bras court du chromosome 4. Les signes cliniques sont secondaires à une destruction progressive de certaines populations neuronales du striatum, les neurones somatostatinergiques et cholinergiques semblant respectés. Le mécanisme moléculaire de l'affection reste hypothétique. Une hyperstimulation par des neurotransmetteurs excitateurs (tels le glutamate) ou une diminution de la résistance de certaines cellules à cette excitation sont des mécanismes qui ont été invoqués.

\section{Marie-Françoise Chesselet}

\section{ADRESSE}

M.-F. Chesselet: Professeur-associé. The medical college of Pennsylvania, Philadel- a chorée de Huntington est une affection héréditaire caractérisée cliniquement par des mouvements anormaux involontaires (chorée), accompagnés d'altérations cognitives et comportementales [1, 2]. Dans la forme la plus commune de la maladie, les premiers symptômes apparaissent entre 30 et 50 ans et évoluent sur une période de 15 à 20 ans vers l'invalidité totale avec démence. La chorée de Huntington est transmise sur le mode autosomique dominant. L'affection est rare (10 pour 100000$)$, mais en raison de son début tardif, souvent après la période de procréation, elle concerne un nombre beaucoup plus large de sujets «à risque» dont un parent ou un autre membre de la famille est atteint.

Il est établi depuis de nombreuses années que l'altération pathologique la plus marquée chez ces malades est une mort neuronale massive, largement localisée au striatum (noyau caudé et putamen), qui conduit à l'atrophie progressive de ces structures cérébrales [3]. Ni les causes, ni les mécanismes de cette dégénérescence neuronale ne sont connus.
Aucun traitement n'a prouvé son efficacité pour ralentir ou prévenir les processus pathologiques, quoique les neuroleptiques, avec lesquels certains malades ont été traités, puissent améliorer certains symptômes. Un certain nombre d'études récentes ont suscité un regain d'intérêt pour cette maladie. En tout premier lieu, grâce à l'apport de la génétique moléculaire, un marqueur de l'ADN situé à proximité du gène responsable de la chorée de Huntington a été identifié [4], ouvrant des perspectives entièrement nouvelles pour le dépistage des sujets «à risque », et rendant vraisemblable l'identification du gène lui-même dans un proche avenir. Parallèlement, la systématisation d'études cliniques, radiologiques (CT-scan, tomographie par émission de positons) et pathologiques concernant un large nombre de sujets atteints et «à risque », a permis d'obtenir des informations nouvelles sur le déroulement de la maladie. Finalement, des recherches expérimentales sur les mécanismes d'induction de la mort cellulaire ouvrent la voie à de nouvelles hypothèses pathogéniques et suggèrent de nouvelles possibilités thérapeutiques. Ces diffé- 
rentes informations vont être exanées successivement.

\section{Identification du gène}

Un progrès décisif pour la localisation du gène de la chorée de Huntington a été réalisé dans le laboratoire de J. Gusella au Massachussets General Hospital de Boston en 1983 [4]. La découverte d'un marqueur chromosomal lié au gène de la chorée de Huntington repose sur l'étude de fragments polymorphiques de l'ADN. Cette approche, qui combine les techniques traditionnelles de linkage* génétique et les techniques modernes d'ADN recombinant, a l'avantage d'utiliser comme marqueurs de régions spécifiques de l'ADN des fragments ne correspondant à aucun gène connu et, par conséquent, de couvrir une large portion du génome sans préjuger de la nature de la protéine codée ou du déficit [5]** La majorité des sujets atteints de la chorée de Huntington sont hétérozygotes pour le gène déficient puisqu'il s'agit d'une maladie dominante rare. Gusella et al. ont cherché une autre caractéristique génétique héritée avec le gène responsable de la chorée suivant une fréquence significative. Si deux gènes sont très proches sur un chromosome, la fréquence de recombinaison entre eux au cours de la méiose est très faible et, par conséquent, ces deux gènes sont hérités ensemble. L'identification d'une région de l'ADN présentant un fort degré de variation séquentielle (polymorphisme) entre individus, et dont une forme est le plus souvent héritée avec le gène défectif dans une famille donnée, permet de localiser le gène défectif dans une région proche de celle de ce marqueur. L'identification d'une telle région de l'ADN est possible grâce à l'utilisation d'enzymes de restriction qui coupent l'ADN en chaque point caractérisé par une séquence donnée, séquence qui est spécifique pour chaque enzyme. Une altération du génome dans une de·ces séquences entraîne

\footnotetext{
* Linkage: liaison génétique (c'est-à-dire transmission concertée de deux gènes, localisés à une distance relativement faible l'un de l'autre sur un même chromosome).

* Voir m/s, suppl. au $n^{\circ} 7$, vol. 3, p. 4 $\mathrm{m} / \mathrm{s} n^{\circ} 8$ vol. 4 , octobre 88
}

l'apparition de fragments de tailles différentes lorsque l'ADN génomique est soumis à l'action de ces nucléases. Ce procédé peut donc être utilisé pour suivre les variations génétiques au sein d'une famille [5]. Le succès de cette approche repose en partie sur l'obtention de cellules provenant d'un nombre suffisant d'individus atteints et non atteints de la même famille. Dans le cas de la chorée de Huntington, un facteur décisif a été l'étude systématique d'une famille de taille exceptionnelle vivant sur les rives du lac Maracaibo au Vénézuela [6]. Ce pedigree comporte environ 4000 individus représentant huit générations avec de nombreux sujets atteints, ayant tous hérité le gène déficient d'un ancêtre commun. L'ADN de 290 individus de cette famille $(65$ atteints et 225 «à risque ») a été analysé. Gusella et al. ont identifié une région de l'ADN du chromosome 4 (G8 ou D4Sl0) héritée avec le gène de la chorée dans cette famille et dans une famille nordaméricaine de plus petite taille [4]. Cette observation permet de localiser le gène de la chorée de Huntington au bras court du chromosome 4 chez l'homme. Il est vraisemblable que la distance entre G8 et le locus de la chorée sur l'ADN soit de plusieurs milliers de bases, rendant l'identification du gène par des méthodes classiques longue et ardue. Néanmoins, les données obtenues permettent de concentrer la recherche de nouveaux marqueurs plus proches du gène sur cette région du génome. L'introduction récente de méthodes d'analyse de longs fragments de l'ADN permettent de prédire que le gène de la chorée de Huntington sera identifié dans un proche avenir. L'aboutissement de ces recherches semble avoir été retardé par des difficultés expérimentales particulières liées à la localisation du gène à la région télomérique du bras court du chromosome 4 [7].

La découverte de marqueurs proches du gène a néanmoins permis d'obtenir un certain nombre d'informations nouvelles importantes. D'une part, l'analyse d'un plus grand nombre de familles a montré que la localisation du gène est homogène [8]. D'autre part, l'analyse de familles appartenant au pedigree vénézuélien a permis d'identifier plusieurs sujets homozygotes. L'étude clinique de ces patients n'a mis en évidence aucune différence significative avec les hétérozygotes, ce qui est très inhabituel pour une maladie humaine à transmission dominante [9]. Finalement, il est maintenant possible, à condition d'avoir accès à l'ADN d'un nombre suffisant de membres de la famille, de déterminer si un sujet «à risque » est porteur ou non du gène déficient. Cette information est de grande valeur pour l'étude clinique, radiologique et, éventuellement, pathologique des stades asymptomatiques de la maladie. L'utilisation de ce test comporte, cependant, des aspects humains et éthiques particulièrement délicats [10]. Puisque la chorée de Huntington est une maladie dominante à pénétrance complète, la présence du gène déficient chez un individu signifie que ce sujet développera tôt ou tard les signes de la maladie. En raison du caractère inexorable et dramatique de la chorée de Huntington, cette information ne peut être donnée à la légère. Même si le sujet est en mesure d'accepter la perspective de la maladie plus tard dans la vie, cette information, si elle ne peut être maintenue strictement confidentielle, risque d'avoir des répercussions dramatiques sur la vie de l'individu (mariage, emploi, assurances, etc.). Il n'est donc pas surprenant que nombre de sujets «à risque » choisissent de ne pas être testés, ou de ne pas connaître les résultats de tests réalisés dans le cadre d'une étude systématique. Il faut souligner que la portée d'un diagnostic prénatal est la même. Savoir que le foetus est porteur du gène, équivaut au diagnostic du parent «à risque ».

\section{Données cliniques et pathologiques}

Les symptômes de la chorée de Huntington sont connus depuis le siècle dernier, mais ce n'est qu'au cours des dernières années que des études systématiques et codifiées portant sur de larges populations ont été entreprises. La création d'échelles diagnostiques codifiées est indispensable pour les études prospectives. L'échelle de Shoulson et Fahn [1 l] est utilisée par plusieurs groupes, en particulier pour une large étude entreprise à l'Université de Rochester 
Figure 1. Représentation schématique des connexions du caudoputamen (CP) mentionnées dans cet article. Les neurones principalement affectés dans la chorée de Huntington sont les neurones efférents du striatum (neurones roses). IIs se projettent vers le pallidum (segments interne et externe) et vers la substance noire (partie compacte: SNc, et réticulaire: SNr). Ces neurones sont GABA-ergiques. De plus, certains des neurones striataux se projetant vers le pallidum externe et vers la substance noire contiennent de l'enképhaline, et certains des neurones se projetant vers le pallidum interne et la substance noire contiennent les tachykinines (substance $P$ et $K)$, et la dynorphine. Des résultats récents suggèrent que les neurones se projetant vers le pallidum externe et la partie réticulaire de la substance noire sont affectés les premiers au cours de la chorée de Huntington. Le striatum contient plusieurs types d'interneurones (neurones blancs à raies noires). Parmi eux, les neurones somatostatinergiques et cholinergiques semblent préservés dans la chorée de Huntington, mais le niveau de choline-acétyltransférase, l'enzyme de synthèse de l'acétylcholine est souvent diminué. Le caudo-putamen reçoit une projection glutamatergique excitatrice du cortex cérébral (neurones rouges). Par analogie avec l'action neurotoxique du glutamate et d'agonistes de ses récepteurs comme l'acide kainique et l'acide quinolinique, il a été postulé que cette voie cortico-striatale pourrait jouer un rôle dans la lésion des neurones du striatum. Comme les autres systèmes afférents au striatum, la voie dopaminergique nigro-striatale (neurones noirs) est préservée dans la chorée de Huntington. Les afférences striatales originaires de l'amygdale et du thalamus ne sont pas représentées pour faciliter la lecture du schéma. (Diagramme exécuté par L.T. Weiss).

et pour l'analyse des familles vénézuéliennes. Un certain nombre de données émergent de ces études et d'autres. En premier lieu, les signes de début ont été examinés avec plus d'attention. Les débuts de chorée marqués par des signes psychiatriques (changement de personnalité, dépression, ou même épisode psychotique) ou par des altérations cognitives sont plus fréquents qu'on

mouvements oculaires sont également souvent observés au début de la maladie [12]. A ces signes de début s'ajoutent bientôt les mouvements choréiques typiques, d'intensité progressive. Avec le temps apparaissent des symptômes parkinsoniens et des dystonies qui peuvent devenir prépondérants aux stades avancés de la maladie [6].

Un des intérêts majeurs de la description de l'évolution des symptômes au cours de la chorée de Huntington est le parallélisme observé avec de récentes études pathologiques codifiées. Vonsattel et ses collègues du Massachussetts General Hospital de Boston ont établi une classification pathologique des anomalies trouvées dans le striatum de sujets décédés à différents stades de la maladie [3]. D'après cette classification, le stade 0 correspond à l'absence de mort neuronale décelable dans le striatum,

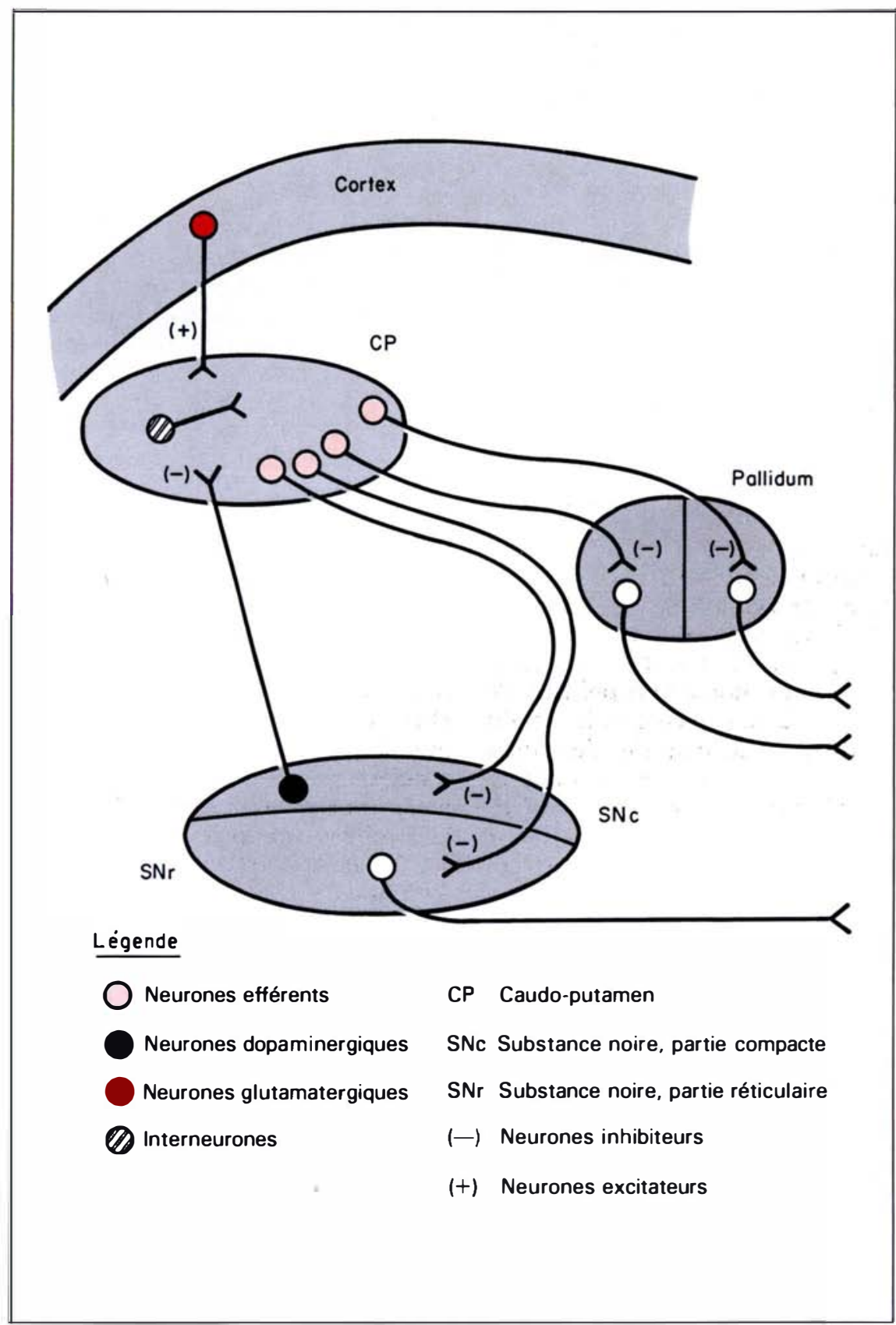

$\mathrm{m} / \mathrm{s} \mathrm{n}^{\circ} 8 \mathrm{vol} .4$, octobre 88 


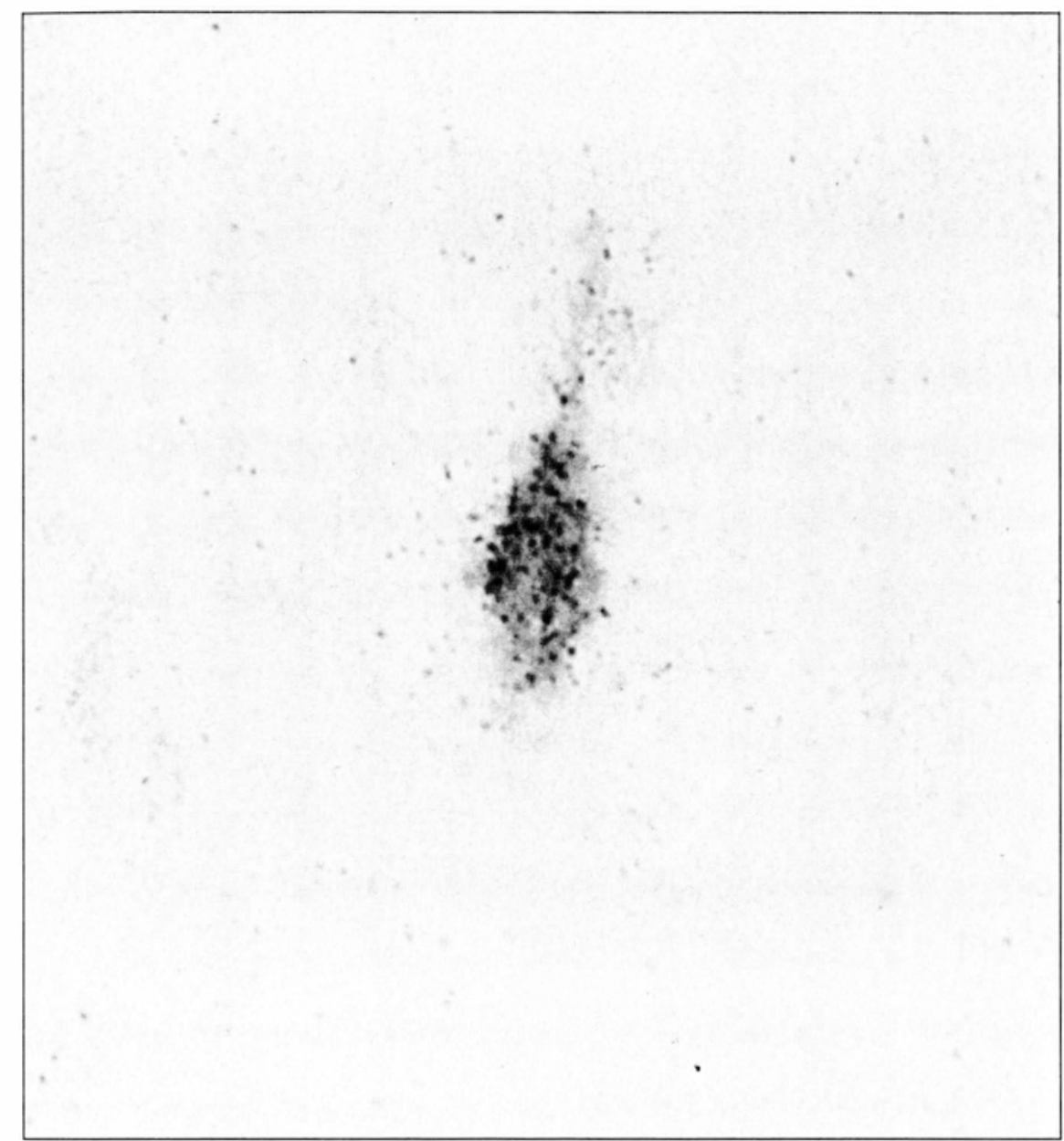

Figure 2. Neurone somatostatinergique du noyau caudé humain. Le taux de somatostatine est élevé dans le caudo-putamen de patients décédés avec le diagnostic de chorée de Huntington. Cette élévation a été attribuée à la survie des interneurones somatostatinergiques du striatum, même à un stade avancé de la maladie. Cette photographie montre un neurone dans une coupe de striatum humain obtenu à l'autopsie. La coloration pourpre du cytoplasme est due à une réaction histologique révélant l'activité de la NADPH-diaphorase, une enzyme caractéristique des neurones somatostatinergiques dans le striatum. Les grains répartis dans le cytoplasme révèlent la présence de l'ARN messager codant pour le précurseur de la somatostatine et détecté par hybridation in situ avec un ARN complémentaire marqué au ${ }^{35} S$ et radio-autographie. (Expérience réalisée par $W$. Stoutenger et M. $-F$. Chesselet).

chez des patients démontrant des signes cliniques non équivoques. Les stades 1 à 4 correspondent à une atteinte progressive du noyau caudé et du putamen, atteinte qui commence dans la région dorso-médiale du noyau caudé et épargne le noyau accumbens, même dans les cas les plus avancés. Cette étude démontre donc que la mort neuronale progresse dans le striatum au cours de la maladie suivant un gradient précis temps tolérés par le malade et par son entourage que les signes moteurs.

La difficulté que l'on rencontre à identifier les premiers symptômes de la chorée de Huntington peut aussi expliquer en partie la controverse soulevée par diverses études de tomographie par émission de positons (PET-scan). Certains groupes ont décelé une diminution de métabolisme dans le striatum chez des sujets "à risque », alors que d'autres pensent que les signes radiologiques apparaissent au même moment que les signes cliniques [13, 14]. Ces différences ont une importance pratique pour le diagnostic des sujets «à risque», et une importance théorique pour la compréhension de l'évolution de la maladie. Au vu des critères de diagnostic utilisés par les différents groupes, il semble que si les anomalies de métabolisme striatal précèdent les signes cliniques, elles ne les précèdent que de peu. Dans certains cas, même, les signes cliniques peuvent précéder les signes radiologiques. Quoiqu'il en soit, les études au PET-scan ont confirmé que le noyau caudé est le premier atteint et que l'atteinte ultérieure du putamen correspond à l'apparition des signes moteurs classiques.

La classification de Vonsattel et al. a aussi été d'une grande utilité pour l'interprétation des études pathologiques les plus récentes. Le but de ces études est de déterminer la chronologie de l'atteinte des différents neuromédiateurs présents dans le striatum au cours de l'évolution de la maladie. Il y a quelques années le groupe de J. Martin, à Boston, a montré une élévation des taux de somatostatine, un neuropeptide présent dans une population d'interneurones du striatum, dans le noyau caudé de sujets atteints [15]. Cette augmentation fut bientôt attribuée à la persistance des neurones somatostatinergiques dans le striatum atteint [8] (figures 1 et 2). Ces résultats étaient particulièrement intéressants parce qu'ils démontraient pour la première fois sans équivoque que toutes les cellules striatales n'étaient pas atteintes au même degré par l'expression du gène déficient. Des études ultérieures ont montré qu'une autre population d'interneurones striataux, contenant le neuromédiateur acétylcholine, 


\section{RÉFÉRENCES}

1. Huntington C; On Chorea. Med Surg Report $1872: 26: 317-21$.

2. Shoulsonl. Huntington's disease. In : Asbury AK, Mckhann CM. McDonald WI. eds. Diseases of the N'en'ous System. Philadelphia : Ardmore Medical Books (W'B Saunders Co), $1986: 1258-67$

3. Vonsattel JP, Meyers RH, Stevens T J, Fer rante RJ, Bird ED, Richardson EP. Neuropathologic classification of Huntington's disease. J N'europathol Exp N'eurol $1985 ; 44$ 5.59-77.

4. Gusella JF, W'exler NS, Conneally PM, et al. A polymorphic DNA marker genetically linked to Huntington's disease. N'ature 1983 $306: 234-9$.

5. Cusella JF, Tanzi RE, Anderson MA, et al. DNA markers for nervous system diseases. Science 1984: 225 : 1320-6.

6. Young AB, Shoulson 1. Penney JB, et al. Huntington's disease in Venezuela : Neurologic features and functional decline. Neurology $1986 ; 36$ : 244-9.

7. Gusella IF Tanzi RE, Bader PI, et al. Deletion of Huntington's disease-linked C;8 (D4SI0) locus in Wolf-Hirschhorn syndrome. Nature $1985 ; 318$ : $75-8$

8. Kowall NW', Ferrante R J. Martin JB. Pat terns of cell loss in Huntington's disease. Trends Neurosci $1987 ; 10: 24-9$.

9. W'exler NS, Y'oung AB, Tanzi RE, el al. Homozygotes for Huntington's disease. Nature 1987 ; 326 : 194-7.

10. Martin JB. Huntington's disease: New approaches to an old problem. N'eurology $1984 ; 34: 1059-72$

11. Shoulson I. Care of patients and families with Huntington's disease. In : Marsden CD Fahn S, eds. Movement Disorders (Int Med Rer Series). London: Butterworth Scientific 1982, 277-90.

12. Bollen E, Reulen JPH, Den Heyer JC, Van der Kamp W, Roos RAC, Buruma OJS. Horizontal and vertical saccadic eye movement abnormalities in Huntington's chorea. J Neurol Sci 1986; 74: 11-22.

13. Mazriotta JC, Phelps ME, Pahl JJ, et al. Reduced cerebral glucose metabolism in asymptomatic subjects at risk for Hunting ton's disease. N'Engl J Med 1987 ; 316 : 357 62.

14. Young AB, Penney JB, Markel DS, Hollingsworth Z. Teener J, Stern J. Cenetic linkage analysis, glucose metabolism, and neurologic examination : comparison in persons at risk for Huntington's disease (HD). Neu-

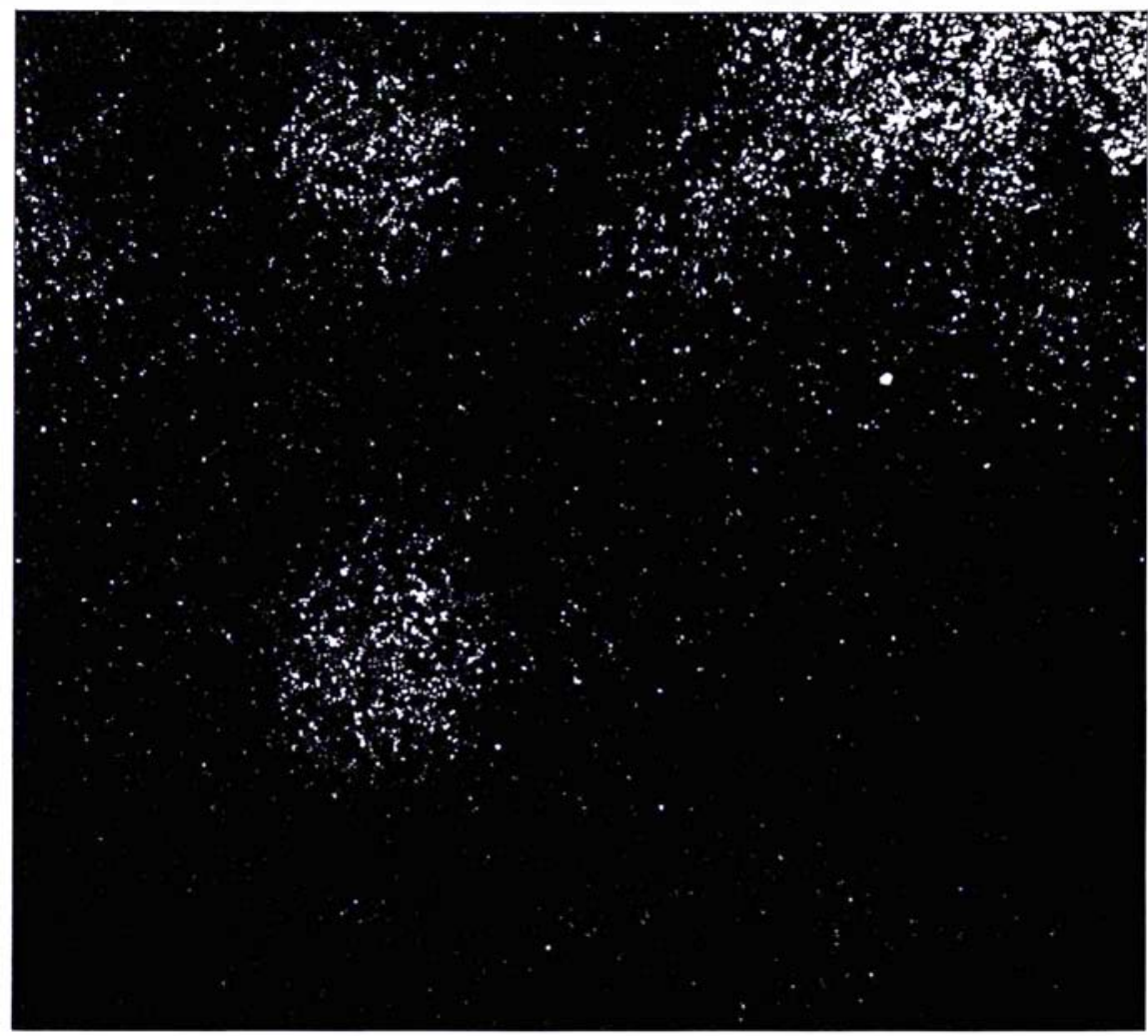

étaient également préservés malgré une fréquente diminution de l'activité de l'enzyme de synthèse de l'acétylcholine, la choline acétyltransférase [8] (figure 1). Donc deux populations d'interneurones, biochimiquement et aussi morphologiquement différents, semblent épargnés. Ceci contraste singulièrement avec le sort des cellules voisines, pour la plupart des cellules efférentes de taille moyenne, qui disparaissent au fur et à mesure de la progression de la maladie (figure 1). Les travaux les plus récents suggèrent cependant que toutes ces cellules efférentes ne sont pas non plus toutes atteintes au même degré. Ceci repose sur l'étude de l'organisation interne du striatum et sur l'analyse des fibres originaires du striatum dans les deux régions innervées par cette structure, le pallidum et la substance noire.

Le striatum est composé de deux "compartiments» imbriqués l'un dans l'autre, la «matrice» et les «striosomes»[16] (figure 3). La matrice striatale est caractérisée par une forte concentration en acétylcholinestérase, l'enzyme responsable de l'hydrolyse de l'acétylcholine. Au sein de cette matrice sont présentes des zones pauvres en acétylcholinestérase, les striosomes. Ces deux compartiments diffèrent dans leurs connexions avec les autres régions du cerveau et dans leurs caractéristiques biochimiques. L'analyse des régions riches et pauvres en acétylcholinestérase dans le striatum de sujets atteints de chorée de Huntington révèle que la distinction entre ces deux compartiments est toujours visible aux stades avancés de la maladie, mais que la taille de la matrice est réduite plus précocement que celle des striosomes [8]. Ceci signifie que les neurones de la matrice sont atteints avant ceux des striosomes. La chorée n'est cependant pas une maladie générale de la matrice striatale puisque les interneurones somatostatinergiques et cholinergiques qui survivent sont situés préférentiellement dans cette région. De plus l'étude immunohistochimique des axones originaires des compartiments striataux suggère que, même au sein de la matrice, il existe des différences dans la susceptibilité des neurones efférents du striatum au processus pathologique. 
Figure 3. Striosomes et matrice. Il a été démontré au cours des dernières années que le striatum est composé d'au moins deux compartiments distincts, les * striosomes vet la * matrice . Ces deux régions striatales sont imbriquées, mais distinctes l'une de l'autre par leurs connexions et des concentrations différentes en nombreux neuromédiateurs et récepteurs. Cette photo illustre une des différences entre striosomes et matrice en montrant la distribution des sites de liaison de la naloxone, un ligand des récepteurs aux opiaces de type $\mu$ dans le striatum du rat. Les zones riches en sites de liaison, révélées par un signal autoradiographique (grains blancs sur la photo) corres. pondent aux « Striosomes $»$ et apparaissent isolées au sein d'une "matrice" non marquée. (Expérience par $M$. F. Chesselet et $E$. Robbins).

Figure 4. Enképhaline (A) et substance $P$ (B et $C$ ) détectées par immunohistochimie dans les terminaisons des neurones efférents du striatum innervant le pallidum interne (GPe), le pallidum externe (GPi) et la substance noire (SN) d'un singe Rhésus. Ces peptides ont la même distribution chez l'homme, et disparaissent au cours de la chorée de Huntington. D'après les travaux de Reiner et al. [19], l'innervation enképhalinergique du pallidum externe (A) et l'innervation à substance $P$ de la substance noire réticulaire $(C)$ disparaissent plus précocément au cours de la maladie que les fibres à substance $P$ innervant le pallidum interne (B). (Expérience réalisée en collaboration avec Ann Graybiel au MIT).

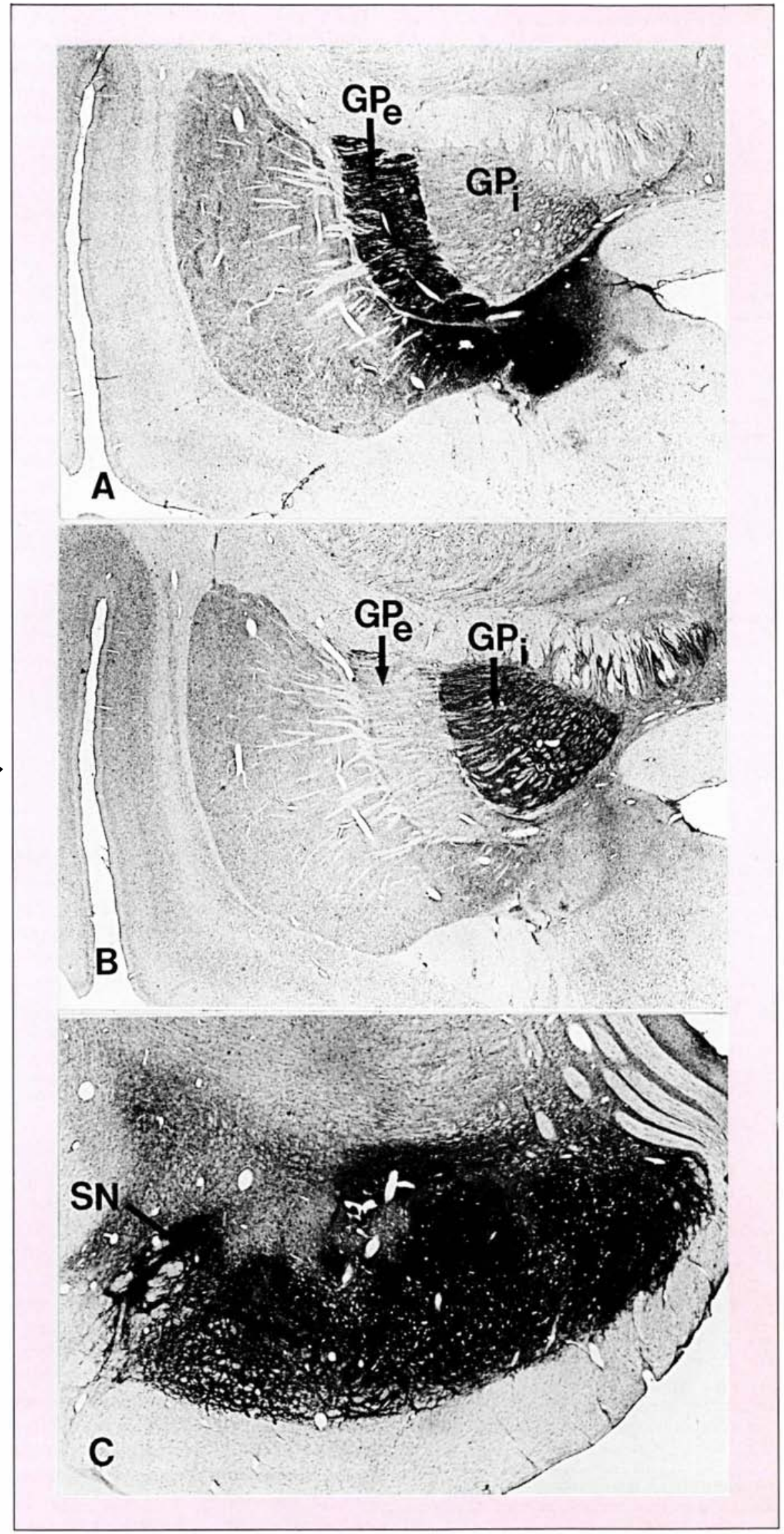




\section{REEEERENCES}

15. Aronin N, Cooper PE, Lorenz LJ, et al. Somatostatin is increased in the basal ganglia in Huntington disease. Ann Neurol 1983 ; 13 : 519-26.

16. Graybiel AM. Neurochemically specified subsystems in the basal ganglia. In : Evarts E, ed. Functions of the Basal Ganglia. Ciba Foundation Symposium 107. London: Pitman, 1984 : 114-44

17. GerfenC. The neostriatal mosaic: I Compartmental organization of projections from the striatum to the substantia nigra in the rat. J Comp Neurol $1985 ; 236$ : 454-76.

18. Graybiel AM. Neuropeptides in the basal ganglia. In : Martin JB, Barchas JD, eds. Neuropeptides in Neurologic and Psychiatric Disease. New York: Raven Press, 1986.

19. Haber $\mathrm{SN}$, Watson $\mathrm{SJ}$. The comparative distribution of enkephalin, dynorphin and substance $P$ in the human globus pallidus and basal forebrain. Neurosci $1985 ; 14$ : 1011 . 24.

20. Reiner A, Albin RL, Anderson KD, D'Amato CJ, Penney JB, Young AB. Differential loss of striatal projection neurons in Huntington's disease. Proc Nall Acad Sci USA (sous presse).

21. Penney JB, Young AB. Striatal inhomogeneities and basal ganglia function. Move ment Disorders 1986: 1:3-15

22. Beal MF, Kowall NW, Ellison DW, Mazurek MF, Swartz KJ, Martin JB. Replication of the neurochemical characteristics of Huntington's disease by quinolinic acid. Nature 1986 ; 321 : 168-7i.

23. Schwarz R, Coyle JT. Striatal lesions with kainic acid : Neurochemical characteristics. Brain Res 1977 ; 127 : 235-49.

24. Boeginan RJ, Smith Y, Parent A. Quinolinic acid does not spare striatal neuropeptide Y-immunoreactive neurons. Brain Res 1987 $415: 178-82$

25. Rothman SM, Olney JW. Excitotoxicity and the NMDA receptor. Trends Neurosci 1987 ; 10 : 299-302.

26. Koh JY, Peters S, Choi DW. Neurons containing NADPH-diaphorase are selectively resistant to quinolinate toxicity. Science $1986 ; 234: 73-6$.

27. Choi DW. Dextrorphan and dextromethorphan attenuate glutamate neurotoxicity.
Des expériences de traçage anatomique chez le rat [17] ont montré que les neurones de la matrice se projettent sur le pallidum externe, le pallidum interne, et la partie réticulée de la substance noire. Les striosomes, au contraire, se projettent sur la partie compacte de la substance noire. Le contenu biochimique des neurones projetant vers ces régions diffère [ 18 , 19]. La projection du striatum vers le pallidum externe contient de l'enképhaline, celle vers le pallidum interne de la substance $\mathbf{P}$ et de la dynorphine, et celle vers la substance noire de l'enképhaline, de la substance $P$ et de la dynorphine (chez les primates) (figure 4). Ces trois projections contiennent aussi du GABA (acide $\gamma$-aminobutyrique) qui coexiste dans certains neurones avec les neuropeptides mentionnés plus haut. A. Reiner et al. viennent de montrer que les fibres enképhalinergiques du pallidum externe, ainsi que les fibres à substance $\mathbf{P}$ innervant la partie réticulée de la substance noire disparaissent précocément au cours de la chorée de Huntington, alors que les fibres à substance $P$ projetant vers le pallidum interne persistent jusqu'à un stade plus avancé de la maladie [20]. Les fibres à substance $P$ innervant la partie compacte de la substance noire semblent les moins atteintes. Ces résultats suggèrent que les neurones de la matrice projetant vers le pallidum externe et la partie réticulée de la substance noire sont les plus vulnérables, alors que la projection vers la partie interne du pallidum l'est moins. Par ailleurs, la projection striosomale vers la partie compacte de la substance noire est relativement préservée.

Une analyse plus détaillée du contenu immunohistochimique de ces projections en fonction de l'évolution de la chorée est bien sûr nécessaire, mais les données obtenues à ce jour permettent déjà de faire un parallèle entre la progression de la mort neuronale et l'évolution des symptômes. Selon Penney et Young, l'atteinte précoce de la projection du striatum vers le pallidum externe expliquerait la prépondérance des mouvements anormaux de type choréique durant les premiers stades de la maladie parce que cette projection joue normalement un rôle dans la suppression des mouvements non désirables [21]. L'atteinte plus tardive de la projection du striatum vers le pallidum interne serait au contraire responsable de l'akinésie et de la rigidité observées après plusieurs années d'évolution. En faveur de cette hypothèse, les mêmes auteurs ont observé une perte précoce des fibres à substance $\mathrm{P}$ dans le pallidum interne chez des sujets chez qui rigidité et akinésie prédominent, en particulier ceux dont la chorée débute dans l'enfance (forme juvénile) [20]. Finalement, l'atteinte de la projection du striatum vers la partie réticulée de la substance noire et la perte de régulation des neurones nigraux projetant vers le colliculus supérieur qui en résulte, expliqueraient la fréquence des anomalies des mouvements oculaires observés au début de la maladie [22].

\section{Hypothèses pathogéniques}

Dans l'attente de l'identification du gène responsable de la chorée de Huntington, les hypothèses pathogéniques sont encore fondées principalement sur les données pathologiques. L'apport majeur des données décrites plus haut est de suggérer que l'atteinte des neurones striataux est progressive, et probablement localisée originellement à une population réduite de neurones. L'atteinte ultérieure d'autres populations neuronales pourrait être due soit à l'extension du mécanisme originel à des cellules moins vulnérables, soit à des mécanismes transsynaptiques.

Deux mécanismes principaux sont habituellement évoqués pour expliquer la mort cellulaire dans le cerveau : la présence d'une toxine neuronale ou l'absence d'un facteur trophique. De nombreux groupes s'intéressent à l'identification de facteurs trophiques cérébraux mais jusqu'à présent l'hypothèse neurotoxique a dominé la scène en ce qui concerne la chorée de Huntington. Ceci est dû principalement à la découverte, il y a plus de dix ans, de la toxicité de l'acide kaïnique, une substance capable de détruire les corps cellulaires neuronaux au voisinage de son point d'injection dans le cerveau, tout en respectant les axones afférents ou de passage [23]. En raison de ces propriétés, l'acide 
kaïnique injecté dans le striatum reproduit ce qu'on croyait être un modèle de chorée de Huntington. La découverte de la vulnérabilité différente de certains neurones striataux a remis cette assertion en question. Cependant d'autres toxines, en particulier une toxine endogène, l'acide quinolinique, pourraient produire des lésions neuronales plus proches de celles de la chorée, en épargnant les neurones somatostatinergiques du striatum [22]. L'accord n'est pas fait sur ces observations [24] mais le rôle d'une action toxique à l'origine de la mort cellulaire striatale dans la chorée de Huntington suscite toujours beaucoup d'intérêt. Ceci est dû en particulier à la découverte du rôle neurotoxique de l'acide glutamique, un acide aminé excitateur qui serait le neuromédiateur utilisé, entre autres, par les neurones cortico-striataux [25]. Dans des conditions expérimentales adéquates, l'acide glutamique (et l'acide aspartique) peut provoquer la mort neuronale in vivo. De plus, ces acides aminés pourraient jouer un rôle dans certaines morts cellulaires in vivo [25]. En effet, les antagonistes de récepteurs au glutamate (récepteurs au $\mathrm{N}$. méthyl aspartate, ou NMDA) préviennent la mort cellulaire provoquée par un épisode anoxique dans certaines régions cérébrales. Il est possible que le glutamate libéré par les terminaisons cortico-striatales joue un rôle dans la mort neuronale de la chorée d'autant plus que les neurones somatostatinergiques sont peu vulnérables à l'action toxique de cet acide aminé [26] (figure 1). Cette différence de susceptibilité pourrait s'expliquer par un nombre différent de récepteurs au glutamate, ou par la présence de systèmes de protection intraneuronaux : activités enzymatiques ou présence de différentes concentrations de certaines protéines liant le calcium. Des essais thérapeutiques fondés sur l'utilisation d'antagonistes des récepteurs au glutamate sont actuellement en cours [27].

Quelle que soit l'hypothèse pathogénique avancée, il faut expliquer pourquoi la mort cellulaire est pratiquement limitée au striatum. Il est possible que l'expression du gène déficient soit localisée. La découverte récente de gènes multiples à expression régionales (ou de produits mul- tiples d'un même gène par génération de messagers différents), codant pour les récepteurs à un neuromédiateur donné suggère que ce pourrait être le cas. Ou bien c'est la combinaison de plusieurs facteurs qui rendrait ces cellules particulièrement vulnérables, par exemple un déficit en protéines liant le calcium dans un neurone recevant une abondance d'influences excitatrices. Dans ce cas, le gène pourrait être exprimé dans toutes les cellules de l'organisme, ou dans une autre région cérébrale, et influencer le striatum indirectement. Enfin, il faut expliquer pourquoi les symptômes de la chorée de Huntington n'apparaissent que durant la seconde partie de la vie. Nous savons maintenant que la mort cellulaire ne précède pas les signes cliniques. Ceux-ci ne reflètent donc pas l'atteinte d'un «seuil symptomatique». Il est toutefois possible qu'une altération fonctionnelle non décelable par les méthodes actuelles précède la mort neuronale. Quoiqu'il en soit, le début tardif de la chorée de Huntington peut s'expliquer de plusieurs façons. Soit le gène déficient n'est exprimé que tardivement au cours de la vie, soit des facteurs liés au vieillissement de l'organisme, ou plus particulièrement du striatum, déclenchent le processus de mort cellulaire lorsque le gène déficient est absent.

Le fait que la chorée de Huntington soit héritée sur le mode dominant suggère que l'anomalie génétique est due à un gain plutôt qu'à une perte de fonction [11]. Il est possible que l'identification du produit du gène mutant suffise à révéler le mécanisme pathogénique de la maladie. Ce ne sera pas nécessairement le cas et, dans cette hypothèse, l'accumulation des données cliniques et pathologiques sur la chorée ainsi que les données expérimentales sur l'organisation anatomique et fonctionnelle du striatum prendront une importance capitale pour la compréhension et, on peut l'espérer, la prévention et le traitement de cette maladie dévastatrice. Dans tous les cas, il est vraisembable que la démarche développée et les connaissances acquises lors de l'étude de la chorée de Huntington seront utiles pour l'étude d'autres maladies génétiques du système nerveux

\section{Summary}

Huntington's chorea is an autosomal dominant hereditary disease characterized by progressive abnormal movements and dementia. In the most common form, the disease usually appears between 30 and 50 years of age. The late onset of the disease, after procreation age, explains the existence of numerous at risk subjects, children of one affected parent. The recent discovery of a DNA marker close to the affected gene has allowed molecular genetisists to locate the defect to the tip of the short arm of chromosome 4. Thanks to this and other markers, it is now possible to identify affected individuals before the onset of the disease in a number of cases. Coupled with these molecular advances, clinical and radiological studies, as well as pathological studies begin to unveal the progression of the illness, characterized by massive, but selective cell loss, progressing from the dorsomedial region of the caudate nucleus to the putamen.
TIRÉS A PART

M.-F. Chesselet. 